\title{
Interacciones genéticas y genético-ambientales como efecto modificador del riesgo de cáncer de próstata: un diseño «case-only»
}

\author{
D ante Cáceres $\mathrm{L}^{1 \mathrm{a}}$, Jeannette Iturrieta $\mathbf{G}^{\mathbf{2 b}}$, \\ Cristian Acevedo $\mathrm{C}^{3 \mathrm{C}}$, Christian Huidobro $\mathrm{A}^{3}$, \\ Nelson Varela $F^{2 d}$, Mario Escala $Z^{2}$, Luis $Q$ uiñones $\mathrm{S}^{2 \mathrm{e}}$. \\ Gene-gene and gene-environment \\ interactions as modifier factors of \\ prostatic cancer risk: «a case-only» \\ design study
}

Background: The role of susceptibility low penetrance genes and environmental factors in the etiology of prostate cancer (PCa) is unclear, but may involve in some cases multiple alleles at multiple loci. Aim: To evaluate the association of gene-gene and gene-environment interactions with PCa. Patients and methods: One hundred three subjects with biopsy proven PCa were studied, using a case-only design. All were interrogated about smoking habits. Polymorphisms for Glutathione-Stransferase (GST) and Cytochrome P4501A1 (CYP1A1), were measured in DNA extracted from peripheral lymphocytes, using a restriction fragment length polymorphism analysis. Results: Our findings suggest that gene-gene interactions between GSTT1 and CYP1A1 high risk genotypes were positive modifiers and had a high predictive value for the presence of PCa, compared with non-susceptibility genotypes. The interaction between susceptibility genotypes and smoking did not modify the risk for PCa. Conclusions: Gene-gene interactions may play a role modulating the susceptibility to PCa in a proportion of affected individuals (Rev Méd Chile 2004; 132: 961-66).

(Key Words: Cytochrome P-450 CYP1A1; Glutathione S-transferase; Polymorphism (Genetics); Prostatic neoplasms)

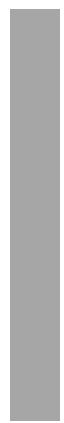
Recibido el 27 de enero, 2004. Aceptado en versión corregida el 29 de junio, 2004.
Trabajo financiado por Proyecto Fondecyt \# 3020043 (Postdoctoral), Corporación Nacional del Cáncer.
${ }^{1}$ División de Salud Ambiental y Ocupacional, Escuela de Salud Pública, Facultad de Medi- cina, Universidad de Chile. 'Laboratorio de Carcinogénesis Química y Farmacogenética, Programa de Farmacología Clínica y Molecular, ICBM, Facultad de Medicina, Universidad de Chile. ${ }^{3}$ Corporación Nacional del Cáncer (CONAC), Santiago, Chile.
amédico Veterinario, Magíster en Salud Pública
bBioquímica
CMagíster en Ciencias Médicas
dTecnólogo Médico, Magíster en Bioquímica
eBioquímico, PhD

Correspondencia a: Dr. Luis Quiñones Sepúlveda. Teléfono: 6786376. Fax: 7372783. E mail: lquinone@med.uchile.cl 
$\mathrm{D}$ e los cánceres diagnosticados en el hombre, el cáncer de próstata $(\mathrm{CaP})$ es uno de los más comunes después del cáncer de piel y la segunda causa de muerte en los hombres después del cáncer pulmonar. Este, raramente ocurre antes de los 55 años de edad y la mayoría de los casos se diagnostican sobre los 65 años. Se estima que el $\mathrm{CaP}$ ha aumentado aproximadamente $70 \%$ en los últimos 30 años, probablemente explicado por factores tales como la detección precoz y aumento en la esperanza de vida, entre otros ${ }^{1}$. En Chile, la mortalidad por $\mathrm{CaP}$ ocupa el cuarto lugar en la mortalidad general por cáncer, después de cáncer gástrico, de pulmón y de mama, y el segundo lugar como causa de muerte entre los varones, similar a lo que acontece en muchas regiones del mundo ${ }^{2}$. De acuerdo a antecedentes de la Corporación Nacional del Cáncer (CONAC), se estima que aproximadamente 1.500 varones mueren anualmente por esta enfermedad en Chile ${ }^{3,4}$.

Recientes estudios indican que factores genéticos o ambientales, tales como antecedentes familiares de CaP (el padre, el hermano, el tío), étnicos o raciales, la edad, la alimentación y los estilos de vida jugarín un rol decisivo en la etiología de esta enfermedad ${ }^{1,5-7}$. Lichtenstein y cols, en un estudio realizado en gemelos y mellizos, concluyeron que el factor genético explicańa aproximadamente $42 \%$ del niesgo de $\mathrm{CaP}$, el resto estańa explicado por factores ambientales ${ }^{8}$. El papel de los factores ambientales ha sido ampliamente sugerido en la etiología de $\mathrm{CaP}$, ya que intemacionalmente la variabilidad observada en la incidencia difiere en un orden de hasta 30 veces, y también por el hecho de que las tasas de esta enfermedad en los inmigrantes tienden a igualarse con la de los países anfitriones ${ }^{9}$. Entre estos factores, podemos considerar la exposición a químicos ambientales (xenobióticos), tales como los compuestos presentes en emisiones diesel o de humo de tabaco, los que son biotransformados en el organismo por complejos enzimáticos celulares en metabolitos que pueden llegar a ser altamente reactivos 0 inertes. La relación entre los genes que codifican para estas enzimas, la exposición a xenobióticos ambientales y su asociación con el resgo de cáncer ha sido extensivamente estudia$\mathrm{da}^{10,11}$. Muchos de estos genes son polimónficos y, por lo tanto, pueden tener profundos efectos en aumentar o reducir las capacidades metabólicas de las enzimas contribuyendo a la susceptibilidad diferencial a riesgo de cáncer por exposición ambiental ${ }^{12,13}$.
Múltiples artículos se han escrito acerca de las interacciones genéticas, genético-ambientales y su relación con la susceptibilidad a diferentes tipos de cáncer, especialmente sobre polimorfismos en genes de baja penetrancia que codifican para determinadas vías metabólicas en el organismo10,14,15. Sin embargo, una de las principales restricciones, al momento de hacer estos estudios, es el tamaño de la muestra ${ }^{16,17}$. En general, para que estos estudios tengan la potencia adecuada para encontrar asociaciones estadísticas se requieren grandes tamaños de muestra, que en el caso de enfermedades de baja incidencia puede ser un factor limitante. Se estima que para evaluar interacciones, el tamaño de la muestra debería ser, a lo menos, cuatro veces el que se necesitaría para valorar sólo los efectos principales ${ }^{18}$. Considerando estos antecedentes y la creciente información que está generando el proyecto Genoma Humano, se han desarrollado metodologías de análisis, que permiten estimar efectos de una manera confiable con tamaños de muestra menores. Uno de los diseños que está siendo usado es el case-only, una variación del típico diseño de casos y controles en el cual se trabaja sólo con los casos. Su principal ventaja es que se pueden usar muestras pequeñas para estimar el efecto conjunto, ya sea, de factores genéticos o ambientales. Una de sus principales limitantes es que no se puede estimar cada efecto en forma aislada y, por lo tanto, no se puede saber cuánto contribuye cada factor a un riesgo específi$\mathrm{Co}^{19}$. Considerando estos antecedentes nos propusimos evaluar estas interacciones y su efecto modificador en un grupo de 103 casos de cáncer de próstata derivado del estudio recientemente realizado por nuestro grupo 20 , enfocado en el estudio de polimorfismos genéticos asociados con el metabolismo de compuestos xenobióticos. En este estudio se determinó la relación entre los genotipos susceptibles de GST, CYP1A1, la exposición a humo de tabaco y el riesgo de CaP.

\section{Material y MÉTodo}

Diseño del estudio y selección de los pacientes. El diseño utilizado fue una variación del tradicional diseño de casos y controles, denominado caseonly ${ }^{19,21}$. La selección de los pacientes ha sido explicada en detalle en Acevedo y cols ${ }^{20}$. Brevemente, desde 1999 hasta la fecha, la Corporación 
Nacional del Cáncer (CONAC) ha realizado varios programa de screening en la población chilena para pesquisar $\mathrm{CaP}$ en individuos de 45 a 85 años de edad en Santiago, desde donde se obtuvieron las muestras para el presente estudio. El antígeno prostático específico (PSA) y el examen rectal digital (DRE) fueron utilizados como método de tamizaje. Las personas sospechosas (PSA $\geq 4 \mathrm{ng} / \mathrm{dl}$ o DRE alterados, o ambos) fueron sometidas a biopsia e histológicamente confirmados. Finalmente, un total de 103 casos de CaP no relacionados familiarmente fueron incluidos en este estudio. Todos los pacientes firmaron un consentimiento informado bajo un protocolo aprobado por el Comité Ético para los Estudios en Seres Humanos de la Universidad de Chile y basado en la declaración de Helsinki.

Encuesta sobre exposición y características personales. Se aplicó una encuesta que consideró los siguientes antecedentes: hábitos de fumar, ingesta de alcohol, uso de contraceptivos orales u hormonas y antecedentes familiares de cáncer prostático. Para la valoración del hábito tabáquico se usó el «\$moking Index» $(\mathrm{SI})^{22}$, considerándose como fumador a los sujetos con un $\mathrm{SI} \geq 800$. Este se calcula multiplicando el número de cigarillos fumados al día por 365 . Fumadores actuales y pasados al momento del estudio fueron considerados como fumadores.

Se realizó, además, una caracterización étnica del grupo de estudio, a través de la medición de las frecuencias del grupo ABO y el estrato socioeconómico de acuerdo a la metodología de Valenzuela y $\operatorname{cols}^{23}$.

Análisis genotípico. El ADN aislado de linfocitos periféricos se amplificó mediante la técnica de PCR usando partidores específicos que permiten la detección de las variantes alélicas en estudio ${ }^{20,24}$. El ADN amplificado se sometió a digestión con la enzima de restricción Msp1 a $37^{\circ} \mathrm{C}$ por $3 \mathrm{~h}$. Finalmente se determinaron los polimorfismos Msp1 de CYP1A1 y las deleciones en los genes de GSTM1 y GSTT1 a través del análisis de fragmentos de restricción por electroforesis en gel de agarosa (2\%) de acuendo a metodologías previamente descritas $22,24,25$.

Metodología analítica. Las frecuencias genotípicas para GSTM1, GSTT1 y CYP1A1 se calcularon como la proporción de individuos con un genotipo dado dividido por el número total de participantes. Las frecuencias alélicas para CYP1A1 (m2 y m1), fueron calculadas como el número de alelos específicos dividido por el número total de cromosomas. Para analizar si las frecuencias génicas encontradas provienen de una población en equilibrio se calculó la prueba de Hardy-Weinberg, para lo cual se comparó las frecuencias genotípicas observadas y esperadas, utilizando la prueba de $\chi^{226}$. No se observaron diferencias significativas entre las frecuencias genotípicas observadas y esperadas para CYP1A1 (datos no presentados). Los polimorfismos genéticos de GSTM1 y GSTT1 de GST fueron clasificados como nulo (homocigoto delecionado) o presente (heterocigoto $u$ homocigoto para la presencia del gen). La magnitud de la interacción entre estos genotipos y el hábito de fumar con el riesgo de CaP fue evaluado usando una estimación del riesgo relativo «Odds Ratio»(OR) y su correspondiente intervalo de confianza (IC 95\%). Los polimorfismos genéticos y la variable hábito de fumar fueron considerados como factores dicotómicos en el análisis de interacciones genético-ambientales, es decir, considerando en forma conjunta (modelo colapsado) los genotipos $\mathrm{m} 1 / \mathrm{m} 2$ y m2/m2 y como contraparte el genotipo $\mathrm{m} 1 / \mathrm{m} 1$ (Tabla 1). Esto se debe a limitaciones de tipo estadísticas, ya que al

\section{Tabla 1. Tabla de clasificación para la presencia y ausencia de las variantes genotípicas 0 ambientales en un diseño «case-only»}

\begin{tabular}{|cccc|}
\hline Variante & \multicolumn{2}{c|}{$\begin{array}{c}\text { Variante genotípica B } \\
\text { o Factor ambiental }\end{array}$} & $\begin{array}{c}\text { Odds Ratio Case-Only } \\
\left(\mathrm{OR}_{\mathrm{co}}\right)\end{array}$ \\
\hline$(+)$ & $(+)$ & $(-)$ & $\mathrm{ad} / \mathrm{bc}$ \\
$(-)$ & $\mathrm{a}$ & $\mathrm{b}$ & \\
\hline
\end{tabular}

(+): Genotipo susceptible/expuesto; (-): genotipo no susceptible (presente)/no expuesto. 
estratificar por genotipo resultan en celdas con valores nulos o de tamaño insuficiente para evaluar estas interacciones, ya que aumenta significativamente la variabilidad y disminuye notablemente la precisión y la potencia (1-ß) del modelo estadístico para encontrar diferencias significativas. Cada modelo bivariado analizado fue ajustado por la variable confundente edad, para lo cual se utilizó la prueba estadística de Mantel-Haenszel. El efecto de estas interacciones fue estimado utilizando como grupo de referencia aquellos individuos portadores de genotipos considerados de «bajo riesgo», es decir, GST presente, CYP1A1 $\mathrm{m} 1 \mathrm{y}$ no expuestos (no fumadores) ${ }^{21,27,28}$. Todos los análisis estadísticos fueron hechos con el paquete estadístico STATA $7.0^{29}$. Considerando que la pesquisa de estos polimorfismos sería una herramienta más en screening de individuos con riesgo de $\mathrm{CaP}$, determinamos el valor predictivo positivo (VPP), es decir la probabilidad de estar enfermo en pacientes que presentan estos genotipos de riesgo y son fumadores ${ }^{30}$. Este se calculó con la siguiente fórmula:

$$
\begin{aligned}
& \operatorname{VPP}_{(G X G)}=\frac{\operatorname{gen}(+)-\operatorname{gen}(+)}{[\operatorname{gen}(+)-\operatorname{gen}(+)]+[\operatorname{gen}(+)-\operatorname{gen}(-)]} \\
& \operatorname{VPP}_{(\mathrm{GXE})}=\frac{\operatorname{gen}(+)-E(+)}{[\operatorname{ggen}(+)-E(+)]+[\operatorname{gen}(+)-E(-)]}
\end{aligned}
$$

Donde: (+): $\quad$ genotipo susceptible o factor ambiental presente, $\mathrm{y}$

(-): genotipo no susceptible 0 factor ambiental ausente

(GxG): interacción Gene-gene

$(\mathrm{GxE})$ : interacción Gene-ambiente

\section{Resultados}

En la Tabla 2 se presentan las características generales de la muestra de personas con $\mathrm{CaP}$ estudiadas. Como es de esperar el grupo estudiado sobrepasaba significativamente los 55 años, donde la mayoría de los casos de este tipo de cáncer se desarrolla en los varones. El valor promedio del APE fue significativamente mayor que el valor predictivo de $4 \mathrm{ng} / \mathrm{dl}$, sin embargo la dispersión fue muy amplia. El $54 \%$ de los casos dio positivo al tacto rectal. El 20,4\% de los pacientes eran fumadores y 27,8\% reportaron consumo de alcohol. Aproximadamente entre 10\% y $42 \%$ reportó antecedentes familiares de $\mathrm{CaP}$.

En la Tabla 3, se presentan las frecuencias genotípicas para GSTM1, GSTT1 y CYP1A1 en pacientes con cáncer de próstata. El 36,3\% y 6,0\% de los individuos con $\mathrm{CaP}$ presentaron el genotipo susceptible para GSTM1 y GSTT1. Para el caso de CYP1A1 m2, el 61,8\% de los pacientes con $\mathrm{CaP}$ presentaron un genotipo portador de la variante alélica m2. Cuando se estratificó según genotipo, $26,4 \%$ y $55,7 \%$ fueron heterocigotos y homocigotos para la variante genética $\mathrm{m} 2 / \mathrm{m} 2$ y $\mathrm{m} 1 / \mathrm{m} 2$, respectivamente, comparado con el genotipo homocigoto $\mathrm{m} 1 / \mathrm{m} 1$. En la Tabla 4 , se presenta la evaluación de las interacciones entre pares de genotipos estudiados en pacientes con CaP. Como puede observarse, los efectos conjuntos para individuos portadores de genotipos susceptibles para GSTM1-GSTT1 y GSTT1-CYP1A1 presentaron valores de OR (crudos y ajustados) mayores que la unidad, indicando interacción de tipo multiplicativa entre estos genotipos. Esto estaría indicando un riesgo incrementado de $\mathrm{CaP}$ para individuos que presentan ambas características genéticas. En el caso de la GSTM1-CYP1A1 el

\section{Tabla 2. Características generales de los pacientes con cáncer de próstata}

\begin{tabular}{|lcc|}
\hline & $\begin{array}{c}\text { Casos de Ca de próstata } \\
(\mathrm{n}=103)\end{array}$ \\
\hline Antecedentes personales & & \\
$\quad$ Edad (años) $\pm(\mathrm{DE})$ & $68,7 \pm 7,3$ & \\
PSA (ng/dl) $\pm(\mathrm{DE})$ & $35,7 \pm 84,9$ & \\
DRE (+) & 56 & $54,0 \%$ \\
Fumadores (n) & 21 & $20,4 \%$ \\
Consumo de alcohol (n) & 28 & $27,8 \%$ \\
Antecedentes familiares de CaP & & \\
Padre (n) & 10 & $9,7 \%$ \\
Hermano (n) & 10 & $9,7 \%$ \\
Tío paterno (n) & 0 & $0 \%$ \\
Madre (n) & 43 & $41,7 \%$ \\
\hline
\end{tabular}

PSA: Antígeno Prostático Específico; DRE: Examen rectal digital. DE: Desviación estándar. 
Tabla 3. Frecuencias genotípicas para G ST M 1, G ST T 1 y CYP1A1 en pacientes con cáncer de próstata

\begin{tabular}{|c|c|c|c|c|c|}
\hline Genotipo & $\mathrm{n}$ & $\%$ & $\mathrm{n}$ & $\%$ & $\mathrm{p}$ \\
\hline GST & \multicolumn{2}{|c|}{ nulo } & \multicolumn{2}{|c|}{ presente } & \\
\hline GSTM1 & $\begin{array}{l}37 \\
(1)^{x}\end{array}$ & 36,3 & 65 & 63,7 & 0,006 \\
\hline \multirow[t]{3}{*}{ GSTT1 } & $\begin{array}{l}6 \\
(3)\end{array}$ & 6,0 & 94 & 94,0 & $<0,001$ \\
\hline & \multicolumn{2}{|c|}{$\mathrm{m} 2(\mathrm{~m} 1 / \mathrm{m} 2+\mathrm{m} 2 / \mathrm{m} 2)$} & \multicolumn{2}{|c|}{$\mathrm{m} 1(\mathrm{~m} 1 / \mathrm{m} 1)$} & \\
\hline & 63 & 61,8 & 39 & 38,2 & $<0,001$ \\
\hline \multirow{3}{*}{$\begin{array}{l}\text { CYP1A1 } \\
\text { (Msp1) }\end{array}$} & \multicolumn{2}{|c|}{$\mathrm{m} 2 / \mathrm{m} 2$} & & & \\
\hline & 14 & 26,4 & 39 & 73,6 & $<0,001$ \\
\hline & $\begin{array}{l}49 \\
(1)\end{array}$ & 55,7 & 39 & 44,3 & $<0,001$ \\
\hline
\end{tabular}

(): Genotipos no determinados; \%: Frecuencia genotípica; m2: (m1m2 y m2m2); m1: ( m1m1).

Tabla 4. Interacciones genotípicas entre G ST y C YP1A1 y O Rs (crudos y ajustados) en pacientes con cáncer de próstata

\begin{tabular}{|lcccccccc|}
\hline $\begin{array}{l}\text { Combinación de } \\
\text { variantes genotípicas }\end{array}$ & $(++)$ & $(+)$ & $(-+)$ & $(--)$ & ORc & IC 95\% & ORa & IC95\% \\
\hline GSTM1-GSTT1 & 3 & 33 & 2 & 61 & 1,82 & $0,34-9,65$ & 1,48 & $0,36-6,14$ \\
GSTM1-CYP1A1 (m2) & 23 & 14 & 40 & 24 & 0,96 & $0,42-2,22$ & 1,17 & $0,48-2,84$ \\
GSTT1-CYP1A1 (m2) & 4 & 1 & 57 & 37 & 3,14 & $0,34-28,56$ & 4,76 & $0,10-234,5$ \\
\hline
\end{tabular}

ORc: Odds ratio crudo; ORa: Odds Ratio ajustado por edad; (+): genotipo susceptible (nulo); (-): genotipo no susceptible (presente); m2: (m1m2 + m2m2). IC 95\%: intervalo de confianza de 95\%.

valor del OR observado se ajustó a la unidad, indicando una interacción más de tipo aditivo que multiplicativo.

En la Tabla 5, se presenta la evaluación de las interacciones genético-ambientales entre los genotipos estudiados y la exposición al humo de tabaco. Como puede observarse los efectos en conjunto de los genotipos susceptibles GSTM1, GSTT1 y hábito de fumar presentaron valores de OR (crudos y ajustados) menores que la unidad, indicando partidas de interacción menos que multiplicativa. Esto indicaría que el hábito de fumar no incrementaría el riesgo de $\mathrm{CaP}$ a través de estos polimorfismos. En cambio, un efecto de tipo multiplicativo fue observado entre GSTT1 nulo y exposición a humo de tabaco.

En la Tabla 6, se presenta el VPP para las interacciones $\mathrm{GxG}$ y $\mathrm{GxE}$ para las diferentes combinaciones de genotipos y factor ambiental. Las combinaciones de GST con CYP1A1 presenta- 
Tabla 5. Interacciones genético-ambientales entre G ST, CYP1A1, hábito de fumar y 0 Rs (crudos y ajustados) en pacientes con cáncer de próstata

\begin{tabular}{|lcccccccc|}
\hline $\begin{array}{l}\text { Variantes genotípicas y } \\
\text { hábito de fumar }\end{array}$ & $(++)$ & $(+)$ & $(-+)$ & $(--)$ & ORc & IC $95 \%$ & ORa & IC95\% \\
\hline GSTM1-fumar & 6 & 31 & 15 & 50 & 0,64 & $0,22-1,85$ & 0,97 & $0,27-3,51$ \\
GSTT1-fumar & 2 & 4 & 19 & 77 & 2,02 & $0,34-12,06$ & 2,67 & $0,30-23,56$ \\
CYP1A1(m2)-fumar & 12 & 52 & 9 & 29 & 0,74 & $0,27-1,98$ & 0,42 & $0,07-2,39$ \\
\hline
\end{tabular}

ORc: Odds ratio crudo; ORa: Odds ratio ajustado por edad; (+): genotipo susceptible (nulo)-fumador; (-): genotipo no susceptible (presente)-no fumador; $\mathrm{m} 2$ : (m1m2 $+\mathrm{m} 2 \mathrm{~m} 2)$. IC 95\%: intervalo de confianza de $95 \%$.

Tabla 6. Valor Predictivo Positivo (VPP) para las interacciones genéticas y genético-ambientales entre GST, CYP1A1, hábito de fumar en pacientes con cáncer de próstata

\begin{tabular}{|lcclcc|}
\hline $\begin{array}{l}\text { Gen-gen } \\
\text { interacción }(\mathrm{GxG})\end{array}$ & $\%$ & IC 95\% & $\begin{array}{l}\text { Gen-ambiente } \\
\text { interacción }(\mathrm{GxE})\end{array}$ & $\%$ & IC $95 \%$ \\
\hline GSTM1-GSTT1 & 8,3 & $2,2-23,6$ & GSTM1-Fumar & 16,2 & $6,8-32,7$ \\
GSTM1-CYP1A1 (m2) & 62,2 & $44,8-77,1$ & GSTT1-Fumar & 33,3 & $6,0-75,9$ \\
GSTT1-CYP1A1 (m2) & 80,0 & $29,9-98,9$ & CYP1A1(m2)-Fumar & 18,8 & $10,5-30,8$ \\
\hline
\end{tabular}

IC: Intervalo de confianza del 95\%; GxG: Gen-gen interacción; GxE: Gen-ambiente interacción. IC 95\%: intervalo de confianza de $95 \%$.

ron un elevado VPP, en cambio, el efecto conjunto de genotipos susceptibles y hábito de fumar presentó un modesto VPP.

\section{DisCUSIÓN}

Las enzimas CYP450 (CYP) metabolizan una amplia variedad de compuestos endógenos y exógenos, tales como los esteroides y xenobióticos ambientales. El gen CYP1A1 codifica para la enzima aril hidrocarburo hidroxilasa $(\mathrm{AHH})$ que se expresa principalmente en hígado, aunque también se ha descubierto en próstata ${ }^{31}$. CYP1A1 activa hidrocarburos aromáticos como el benzo(a)pireno, transformándolos en epóxidos y productos fenólicos mutagénicos y carcinogénicos; por consiguiente, una alta actividad enzimáti- ca puede predisponer a los pacientes al riesgo de cáncer por aumento de los compuestos carcinogénicos a nivel de los tejidos, tales como la próstata y el pulmón $7,32,33$. Tres polimorfismos genéticos han sido ampliamente estudiados Mspl RFLP (CYP1A1*2A), MspI RFLP (CYP1A1*2C) y CYP1A1*334. Las variantes alélicas de este gen (Val y $\mathrm{m} 2$ ) pueden aumentar la susceptibilidad individual al riesgo de cáncer por aumento en la actividad enzimática de $\mathrm{AHH}^{35}$. Por otro lado, la familia de GSTs cataliza la unión de glutatión con numerosos compuestos potencialmente genotóxicos, tales como radicales aromáticos y epóxidos. Las diferencias individuales observadas en la desintoxicación de compuestos químicos como éstos, frecuentemente son el resultado de deleción en los genes que codifican para este grupo enzimático, particularmente la deleción de GSTM1 
y GSTT1 ${ }^{36,37}$. Individuos expuestos a humo de cigarrillo y que han heredado el genotipo susceptible (GSTM1 o GSTT1 nulo) tendrían incrementada la carga corporal de metabolitos reactivos y, por lo tanto, un mayor riesgo de desarrollar cáncer de próstata ${ }^{25,38}$.

En este estudio, en primer lugar examinamos la interacción entre los polimorfismos genéticos GSTM1, GSTT1 y CYP1A1, y posteriormente analizamos la interacción entre éstos y el hábito de fumar, y su asociación con el riesgo de cáncer de próstata. Cuando estos polimorfismos fueron analizados en forma conjunta, los ORs ajustados fueron mayores que 1.0; indicando que entre estos genes existiría una interacción de tipo multiplicativa, es decir, juntos potencian el riesgo de presentar $\mathrm{CaP}$, claramente el efecto conjunto de los genotipos susceptibles GSTT1 nulo y $\mathrm{m} 2$ de CYP1A1 también fue multiplicativo, sin embargo, estos hallazgos no fueron estadísticamente significativos. Esto estaría indicando, que a lo menos uno de estos genes tendría un efecto potenciador del riesgo de $\mathrm{CaP}$, que puede estar enmascarado por la presencia del otro gen, situación que eventualmente se podría evaluar en un diseño de casos y controles con un tamaño de muestra adecuado. A la fecha en la literatura publicada no hemos encontrado diseños del tipo case-only para estos genotipos y su relación con cáncer de próstata. Murata y cols, en estudios (caso-control) realizados en población japonesa informaron de incrementos en el riesgo de CaP (OR 2,3 CI 95\% 1,18-4,48; OR 2,2 CI 95\% 1,12-4,20) cuando ambos polimorfismos genéticos estuvieron presentes (GSTM1 nulo y CYP1A1 m2) ${ }^{39,40 . ~ P o r ~ o t r o ~ l a d o, ~}$ diversos autores han evaluado polimorfismos genéticos combinados de GSTM1, GSTT1 y riesgo de $\mathrm{CaP}$. En general, no se han reportado asociaciones significativas entre combinaciones de los genotipos susceptibles para GSTM1, GSTT1 y riesgo de $\mathrm{CaP}^{25,31}$. Steinhoff y cols, en cambio, reportaron una significativa asociación entre estos polimorfismos y $\mathrm{CaP}^{38}$.

Cuando evaluamos modelos bivariados de interacciones genético-ambientales y riesgo de $\mathrm{CaP}$, se observó un efecto multiplicativo no significativo entre el genotipo susceptible para GSTT1 y hábito de fumar. Las otras interacciones evaluadas presentaron valores menores que 1.0; indicando partidas de interacción de tipo no-multiplicativo.
Esto estaría indicando que el hábito de fumar y estos polimorfismos genéticos serían factores independientes en la etiología de cáncer de próstata. Escasos estudios han evaluado directamente estas interacciones. Kelada y cols ${ }^{41}$ comunicaron un incremento en la probabilidad de cáncer de próstata en hombres fumadores y portadores del genotipo no delecionado de GSTT1, comparado con no fumadores portadores del genotipo susceptible. En cambio, los mismos autores no encontraron relación entre GSTM1 susceptible genotipo, hábito de fumar y riesgo de $\mathrm{CaP}$. Similares hallazgos comunican Murata y $\operatorname{cols}^{40}$, quienes no observaron ningún efecto modificador en $\mathrm{CaP}$ cuando el hábito de fumar fue considerado. En general no existe clara evidencia epidemiológica que apoye una relación causal entre el hábito de fumar y riesgo de $\mathrm{CaP}^{2,42,43}$. Un estudio recientemente publicado, sin embargo, indica que individuos con un alto consumo de cigarrillos, aumentarían su riesgo de desarrollar $\mathrm{CaP}$, especialmente en formas más agresivas de la enferme$\operatorname{dad}^{44}$. Cuando se evaluó el valor predictivo de estos genotipos de riesgo en forma combinada en pacientes de $\mathrm{CaP}$ fumadores y no fumadores, se pudo observar un VPP elevado sólo para las combinaciones genéticas. Este confirma el hecho, que el hábito de fumar no tendría un claro valor predictivo en este tipo de cáncer. Sin embargo, se deben realizar más estudios que confirmen estos hallazgos, especialmente ver el efecto de los diferentes genotipos en forma aislada, para lo cual se requiere un tamaño de muestra significativamente mayor, que permita estratificar en menores grupos sin pérdida de precisión y potencia estadística del estudio.

En la interpretación de estos resultados debemos considerar algunas restricciones que se han descrito en este tipo de análisis; este diseño sólo permite evaluar partidas de interacción de tipo multiplicativa ${ }^{21}$, esto es básicamente debido a que no se puede determinar cada efecto por separado, ya que no se cuenta con un grupo control. Sin embargo, este tipo de diseño entrega una mayor precisión que el tradicional diseño de casos y controles, ya que el tener un grupo control aumenta la variabilidad y la probabilidad de cometer diferentes tipos de sesgos. Lo más difícil en un estudio de casos y controles es elegir un buen grupo control. Otro factor es que los genes 
bajo estudio deben segregar independientemente, no estar ligados en un mismo cromosoma, o tener diferentes frecuencias génicas en la población estudiada. Los genes estudiados en esta investigación se encuentran ubicados en diferentes cromosomas y, por lo tanto, este supuesto se cumpliría en nuestro estudio. Con respecto a la posibilidad de estratificación en este estudio sería at minimum ya que la población estudiada no refirió antecedentes étnicos diferenciales. Además, las frecuencias génicas observadas para estos polimorfismos concuerdan con otros estudios hechos en la población chilena ${ }^{22,45}$. Respecto a este tipo de sesgo de confusión, Wacholder y cols indican que autorreportar la procedencia étnica o racial puede ser la mejor y más apropiada herramienta para reducir el efecto de confusión en estudios sobre cáncer, donde el efecto de estratificación puede ser un factor importante ${ }^{46}$. Otra fuente de

\section{REFERENCIAS}

1. GanN PH. Risk Factors for Prostate Cancer. Reviews in Urology 2002; 4 (Suppl 5): S3-S10.

2. Signorelo L, Adami H. Prostate Cancer. In: HansOlov Adami DH, Dimitrios Trichopoulos, ed. Textbook of Cancer Epidemiology. New York: Oxford University Press 2002; 400-28.

3. INE. Anuario de Estadísticas Vitales. Instituto Nacional de Estadísticas, Gobierno de Chile, Ministerio de Salud, Servicio de Registro Civil e Identificación 2002; 246.

4. CONAC. Web Page www.conac.cl. Vol. Junio de 2004, 2004.

5. Verhage BA, Kiemeney LA. Inherited predisposition to prostate cancer. Eur J Epidemiol 2003; 18: 1027-36.

6. Nelson WG, De Marzo AM, Isaacs WB. Prostate cancer. N Engl J Med 2003; 349: 366-81.

7. Coughun SS, HaLl IJ. A review of genetic polymorphisms and prostate cancer risk. Ann Epidemiol 2002; 12: 182-96.

8. Luchtenstein P, Holm NV, Verkasalo PK, Iluadou A, Kaprio J, Koskenvuo M et aL. Environmental and heritable factors in the causation of cancer analy- sesgo podría acontecer por la forma en que fueron reclutados los individuos en este estudio, ya que es posible que en este grupo haya diferentes grados de evolución de la enfermedad y por lo tanto el efecto de sobrevivencia interfiera en la interpretación de los resultados. Finalmente otros factores no considerados en este estudio, serían la dieta y la exposición ocupacional ${ }^{1,47}$.

En conclusión, nuestros resultados sugieren que en las interacciones genotípicas estudiadas, la presencia de las variantes susceptibles de GST y CYP1A1 tendrían un efecto modificador sobre el cáncer de próstata. En contraste, el efecto de la exposición a humo de tabaco sobre los polimorfismos estudiados no modificó el riesgo de cáncer de próstata. La magnitud y dirección de las modificaciones observadas debieran ser definidas y complementadas en un estudio de casos y controles que consideren un tamaño de muestra adecuado para evaluar interacciones genético-ambientales.

ses of cohorts of twins from Sweden, Denmark, and Finland. N Engl J Med 2000; 343: 78-85.

9. HAYES RB. Gene environment interrelations in prostate cancer. Epidemiol Rev 2001; 23: 163-7.

10. VINEIS P. The relationship between polymorphisms of xenobiotic metabolizing enzymes and susceptibility to cancer. Toxicology 2002; 181-182: 457-62.

11. Nebert DW, Mckinnon RA, Puga A. Human drug metabolizing enzyme polymorphisms: effects on risk of toxicity and cancer. DNA Cell Biol 1996; 15: 273-80.

12. Carmella SG, Borukhova A, Akerkar SA, Hecht SS. Analysis of human urine for pyridine-N-oxide metabolites of 4-(methylnitrosamino)-1-(3-pyridyl)-1-butanone, a tobacco-specific lung carcinogen. Cancer Epidemiol Biomarkers Prev 1997; 6: 113-20.

13. Daly AK, Fairbrother KS, Smart J. Recent advances in understanding the molecular basis of polymorphisms in genes encoding cytochrome P450 enzymes. Toxicol Lett 1998; 102-103: 143-7.

14. El-Zein R, Conforti-Froes N, Au WW. Interactions between genetic predisposition and environmental toxicants for development of lung cancer. Environ Mol Mutagen 1997; 30: 196-204. 
15. Kelada SN, Eaton DL, Wang SS, Rothman NR, KHOURY MJ. The role of genetic polymorphisms in environmental health. Environ Health Perspect 2003; 111: 1055-64.

16. Gauderman WJ. Sample size requirements for association studies of gene-gene interaction. Am J Epidemiol 2002; 155: 478-84.

17. BRENNAN P. Gene environment interaction and aetiology of cancer: what does it mean and how can we measure it? Carcinogenesis 2002; 23: 381-7.

18. Smith PG, DaY NE. The design of case-control studies: the influence of confounding and interaction effects. Int J Epidemiol 1984; 13: 356-65.

19. Yang Q, Khoury MJ, Sun F, Flanders WD. Caseonly design to measure gene-gene interaction. Epidemiology 1999; 10: 167-70.

20. Acevedo C, Opazo JL, Huidobro C, Cabezas J, ITURRIETA J, Quinones L. Positive correlation between single or combined genotypes of CYP1A1 and GSTM1 in relation to prostate cancer in Chilean people. Prostate 2003; 57: 111-7.

21. KHouRY MJ, FLANDERS WD. Nontraditional epidemiologic approaches in the analysis of gene environment interaction: case-control studies with no controls. Am J Epidemiol 1996; 144: 207-13.

22. Quiñones L, Berthou F, Vareia N, Simon B, Gil L, LuCAS D. Ethnic susceptibility to lung cancer: differences in CYP2E1, CYP1A1 and GSTM1 genetic polymorphisms between French Caucasian and Chilean populations. Cancer Lett 1999; 141: 167-71.

23. Valenzuela CY, Acuna MP, Harb Z. Sociogenetic gradient in the Chilean population. Rev Méd Chil 1987; 115: 295-9.

24. Ambrosone CB, Freudenheim JL, Graham S, Marshall JR, Vena JE, Brasure JR et al. Cytochrome P4501A1 and glutathione S-transferase (M1) genetic polymorphisms and postmenopausal breast cancer risk. Cancer Res 1995; 55: 3483-5.

25. Kawajiri K, NaKachi K, Imai K, Yoshi A, Shinoda N, WatanaBe J. Identification of genetically high risk individuals to lung cancer by DNA polymorphisms of the cytochrome P450IA1 gene. FEBS Lett 1990; 263: 131-3.

26. Korf B. Human Genetics: A Problem Based Approach. Second Edition ed, 2000.

27. Вотто LD, Kноury MJ. Commentary: facing the challenge of gene-environment interaction: the two by four table and beyond. Am J Epidemiol 2001; 153: 1016-20.
28. YANG Q, Khoury MJ. Evolving methods in genetic epidemiology. III. Gene environment interaction in epidemiologic research. Epidemiol Rev 1997; 19: 33-43.

29. STATA. STATA Corporation. Intercooled STATA for Windows. 7 ed, 2000.

30. Fietcher R, Fietcher S, EH W. Chapter 3. Diagnosis. In: Satterfield TS, ed. Clinical Epidemiology. The Essentials. Baltimore, Maryland 21201-2436. USA: Willims \& Wilkins, 1996; 43-74.

31. Agundez JA, Martínez C, Olivera M, Galaardo L, Ladero JM, Rosado C et al. Expression in human prostate of drug and carcinogen metabolizing enzymes: association with prostate cancer risk. Br J Cancer 1998; 78: 1361-7.

32. Plummer SJ, Conti DV, Paris PL, Curran AP, Casey G, WrTTE JS. CYP3A4 and CYP3A5 genotypes, haplotypes and risk of prostate cancer. Cancer Epidemiol Biomarkers Prev 2003; 12: 928-32.

33. Shimada T, Yun CH, Yamazaki $H$, Gautier JC, BeAune PH, Guengerich FP. Characterization of human lung microsomal cytochrome P-450 1A1 and its role in the oxidation of chemical carcinogens. Mol Pharmacol 1992; 41: 856-64.

34. Taiol E, Trachman J, Chen X, Toniolo P, Garte S. A CYP1A1 restriction fragment lengh polymorphism is associated with breast cancer in AfricanAmerican women. Cancer Res 1995; 55: 3757-8.

35. HiRvonen A. Combinations of susceptible genotypes and individual responses to toxicants. Environ Health Perspect 1997; 105 Suppl 4: 755-8.

36. Seidegard J, Vorachek WR, Pero RW, Pearson WR. Hereditary differences in the expression of the human glutathione transferasa active on transsteilvene oxide are due to a gene deletion. Proc Natl Acad Sci USA 1988; 85: 7293-7.

37. Pemble S, Schroeder KR, Spencer SR, Meyer DJ, Hawer E, Bolt HM et al. Human glutathione Stransferase theta (GSTT1): cDNA cloning and the characterization of a genetic polymorphism. Biochem J 1994; 300 (Pt 1): 271-6.

38. Steinhoff C, Franke KH, Golka K, Thier R, Romer HC, Rotzel C eт AL. Glutathione transferase isozyme genotypes in patients with prostate and bladder carcinoma. Arch Toxicol 2000; 74: 521-6.

39. Murata M, Shiraishi T, Fukutome $K$, Watanabe $M$, Nagao M, Kubota Y et al. Cytochrome P4501A1 and glutathione S-transferase M1 genotypes as risk factors for prostate cancer in Japan. Jpn J Clin Oncol 1998; 28: 657-60. 
40. Murata M, Watanabe M, Yamanaka M, Kubota Y, Ito $\mathrm{H}$, NagaO M ET aL. Genetic polymorphisms in cytochrome P450 (CYP) 1A1, CYP1A2, CYP2E1, glutathione S-transferase (GST) M1 and GSTT1 and susceptibility to prostate cancer in the Japanese population. Cancer Lett 2001; 165: 171-7.

41. Kelada SN, Kardia SL, Walker AH, Wein AJ, MaLKowicz SB, REBBECK TR. The glutathione S transferase $\mathrm{mu}$ and theta genotypes in the etiology of prostate cancer: genotype environment interactions with smoking. Cancer Epidemiol Biomarkers Prev 2000; 9: 1329-34.

42. LeVI F, La VeCCHIA C. Tobacco smoking and prostate cancer: time for an appraisal. Ann Oncol 2001; 12: 733-8.

43. Sommer F, Klotz T, Schmitz-Drager BJ. Lifestyle issues and genitourinary tumours. World J Urol 2003.

44. Piaskon LA, Penson DF, Vaughan TL, Stanford JL. Cigarette smoking and risk of prostate cancer in middle aged men. Cancer Epidemiol Biomarkers Prev 2003; 12: 604-9.

45. QuiÑones L, LuCAs D, Godoy J, CÁceres D, Berthou F, Varela N et al. CYP1A1, CYP2E1 and GSTM1 genetic polymorphisms. The effect of single and combined genotypes on lung cancer susceptibility in Chilean people. Cancer Lett 2001; 174: 35-44.

46. Wacholder S, Rothman NR, Caporaso N. Counterpoint: Bias from Population Stratification Is Not a Major Threat to the Validity of Conclusions from Epidemiological Studies of Common Polymorphisms and Cancer. Cancer Epidemiology, Biomarkers \& Prevention 2002; 11: 513-20.

47. Seidier A, Heiskel $H$, Bickeboler R, Elsner G. Association between diesel exposure at work and prostate cancer. Scand J Work Environ Health 1998; 24: 486-94.

\section{Agradecimientos}

Los autores desean agradecer la colaboración de la Sra. Catalina Agosin, Directora de la CONAC, por su apoyo a esta investigación. A la Ellison Medical Foundation-Fellowship Program, a través de la International Nutrition Foundation, Inc en Boston, Massachusetts y al Departamento de Epidemiología de la Escuela de Salud Pública de la Universidad de Carolina del Norte, Chapel Hill, USA, por el apoyo y colaboración para el diseño estadístico-epidemiológico durante la estadía del Dr. Dante Cáceres. 